\title{
Correlation between medication errors with job satisfaction and fatigue of nurses
}

\author{
Shahnaz Bolandianbafghi ${ }^{1}$, Tahere Salimi ${ }^{2}$, Maryam Rassouli ${ }^{3}$, Reza Faraji ${ }^{4}$, Mohammadtaghi \\ Sarebanhassanabadi ${ }^{5}$
}

${ }^{1}$ M.Sc., Department of Nursing, Yazd Branch, Islamic Azad University, Yazd, Iran

${ }^{2}$ M.Sc., Department of Pediatrics, Faculty of Nursing \& Midwifery, Shahid Sadoughi University of Medical Sciences, Yazd, Iran

${ }^{3}$ Ph.D., Assistant Professor, Nursing \& Midwifery School, Shahid Beheshti University of Medical Sciences,

Tehran, Iran

${ }^{4} \mathrm{Ph} . D$. Student, Kermanshah Cardiovascular Research Centre, Kermanshah University of Medical Sciences, Kermanshah, Iran

${ }^{5}$ Ph.D. Student, Yazd Cardiovascular Research Center, Shahid Sadoughi University of Medical Sciences, Yazd, Iran

\section{Type of article: Original}

\begin{abstract}
Background: Medication errors which can cause several medical malfunctions, have been in the spotlight recently. Job satisfaction and fatigue seems to be related to the increase of medication errors in nursing.

Objective: This study aimed to determine the correlation between the amount and type of medication errors with job satisfaction and fatigue of nurses working in Shahid Sadoughi hospital in Yazd-Iran.

Methods: This is a descriptive study by a cross-sectional and correlational approach conducted on all the nurses working in an educational hospital who have characteristics of samples. Participants were 170 nurses working in Shahid Sadooghi hospital in Yazd, Iran in 2012. The questionnaires of demographic characteristics, medication administration error and nurses' job satisfaction and fatigue by purposive sampling were used for gathering data. Validity and reliability were checked for internal consistency and stability. Data were analysed by SPSS version 17 using Pearson correlation coefficient and Independent-samples t-test.

Results: The findings did not illustrate a significant relationship between job satisfaction and fatigue with medication errors. Among the demographic characteristics, age of nurses was significantly associated with the frequency of medication errors $(p=0.037)$. However, others were not significantly correlated $(p>0.05)$.

Conclusion: In spite of the findings, based on no significant statistical relationship between job satisfaction and fatigue with the type and amount of medication errors in nursing, the effect of these variables on medication errors cannot be disregarded; thus further studies in this area are suggested.

Keywords: Medication errors, Job satisfaction, Fatigue, Nurse
\end{abstract}

\section{Introduction}

Despite development and improvement in health care facilities, medical errors have occurred more and more in recent years (1). Unfortunately, a large number of these errors consist of nurse's errors while providing care and medication (2). On the other hand, patients' safety and preventing errors and malfunctions are one of the most important objectives of health care systems $(3,4)$. An investigation conducted in 21 hospitals in the Netherlands showed that approximately $6 \%$ of 1.3 million hospitalizations in 2004 were associated with inadvertent harm to the patient (5). Also, complications of medication errors in America, leads to over 177 billion dollars in costs annually (6). Medication errors in Iran do not seem to be lower than similar errors and malpractices in developed countries' health care systems, although there are no rational and accurate statistics for it in our country (7). But according to

\section{Corresponding author:}

Assistant Professor Dr. Maryam Rassouli, Nursing \& Midwifery School, Shahid Beheshti University of Medical Sciences, Tehran, Iran. Tel: +989132586853, Fax:+983535231421, Email: rassouli.m@gmail.com

Received: April 22, 2017, Accepted: June 29, 2017, Published: August 2017

iThenticate screening: June 28, 2017, English editing: July 26, 2017, Quality control: August 12, 2017

This article has been reviewed / commented by three experts

(C) 2017 The Authors. This is an open access article under the terms of the Creative Commons Attribution-NonCommercialNoDerivs License, which permits use and distribution in any medium, provided the original work is properly cited, the use is non-commercial and no modifications or adaptations are made. 
the Ministry of Health, in medical treatment and medical education, billions of dollars are spent each year on maintaining and caring for patients, looking for drug errors and the complications arising from them. The increase in referral cases and complaints from people to the medical system is one of the symptoms of this claim (8). Research shows that the majority of reported mistakes are made by nurses. But this report only contains $25-63 \%$ of the errors. Failure to report an error can occur due to failure to recognize the error (9). But the most important reason is the nurses' fear of punishment and accusation from supervisors and nursing directors for mistakes when administering drugs (10) Medication errors in nursing, particularly from deviation of physicians' orders have many sources including errors in monitoring, administering and calculating drugs, which are all preventable (11). Since medication administration is the most important role of nurses, recurring mistakes and errors in this field are more considerable and also have adverse consequences for patients. However, nurses have a very important role in preventing errors as well as reporting them. Hence, standard protocols and sufficient knowledge are needed for safety of the medication administration process $(12,13)$. Job satisfaction seems to affect nurses' caring performance and patient safety (14). Job satisfaction is important to study for two reasons: First, the human aspect that means employees deserve to be treated fairly and respectfully. Second, the behavioral aspect that means consideration of job satisfaction, can lead employees to behave in a manner that affects their function and organizational duties and helps them to show positive behavior (15). Job satisfaction is an emotional state that is the result of evaluating the job or job experiences (16). Job satisfaction represents a positive or negative attitude towards the job and is influenced by several factors (17). It appears that nurses who are more successful in taking care of patients have higher job satisfaction (18). Due to lack of job satisfaction, in the long term, the organization system is disrupted, rebellion begins, and responsibility is reduced (19). Job dissatisfaction increases stress in nurses, and consequently, the rate of turnover. The materials related to Iranian nurses showed that inadequate conditions, job description, lack of equipment, limited clinical practice, disagreement with doctors and managerial issues, and lack of supporting jobs in nurses, led to job dissatisfaction (20). All these factors go hand in hand to increase medication errors and patients' dissatisfaction, and reduce the quality of care $(21,22)$. Several factors contribute to medication errors in nursing, some of which include fatigue, increased workload, and the nurse's inadequate knowledge of the drug (14). In recent years, the role of fatigue in the incidence of medication errors have been highlighted more. Fatigue, feelings of weakness and weariness of mind which, between them, may interfere with one's ability and role-playing activities is considered. In fact, fatigue is a condition in which the person is unable to detect the continuous feeling of physical and mental performance analysis, capacity reduction is experienced (22). According to research conducted in America, more than a quarter of nurses work about 12 hours a day and about half of them have more than one job, which can lead to increased fatigue (23). With the increasing number of patients and reduced ratio of nurses to patients, the subsequent increased fatigue in nurses, results in increased error rates (24). According to the inconsistencies between the results of research conducted on the relationship between many factors in nurses with medication errors, this study aimed to investigate the correlation between the amount and type of medication errors with nurses' job satisfaction and fatigue.

\section{Material and Methods}

This descriptive correlational cross-sectional study was conducted in Shahid Sadoughi hospital, Yazd. The study population included all nurses working in Shahid Sadoughi hospital. In this study, a census method was used. They were selected by a purposive sampling method based on this inclusion criteria: 1 , at least one year of experience in a clinical department, and 2, having a bachelor's degree in nursing, and a self-statement of physical and mental health. From our study population, the total number of nurses in this hospital was 290, of which 200 nurses were eligible. However, 10 individuals refused to fill out the questionnaires. Thus 190 questionnaires were distributed, and finally, 170 were completed and returned to the researcher. The questionnaires were distributed during three different shifts and were collected at the following shift. The sampling took one month, from December 4th through January 5th, 2012. For gathering data, the questionnaire of demographic characteristics, medication administration errors, Herzberg's Job Satisfaction and fatigue Questionnaire were used. Demographic characteristics consisted of questions such as age, sex, education, marital status, and employment status of nurses. For collecting data of medication errors, the Medication Administration Error Questionnaire developed by Wakefield et al. (2005) was applied (25). Taheri et al. (2011) translated it into Persian (26). The questionnaire was formed in two parts, non-injectable and injectable medication errors, that were scored in the 5-point Likert scale (very low, low, medium, high and very high) (26). Each person in this questionnaire could receive 0 to 110 , of which 0 indicated non-error and 110 indicated the most errors. In order to measure job satisfaction, the Herzberg questionnaire which included 43 statements on a 4-Point Likert scale was used. This questionnaire was initially designed by Herzberg and then developed by Halden in 1980, and finally completed in 2004 by Chiu and Cherng (27). This questionnaire was used by Monjamed et al. in 2004. They used content validity to assess the validity of the questionnaire. In this regard, the 
views of 10 professors of Tarbiat Modarres, Tehran, Iran and Shahid Beheshti Universities are taken into consideration regarding the instruments and the necessary changes regarding the instruments are considered by the professors (17). Each participant in this questionnaire could receive 0 to 172, of which 0 indicated complete dissatisfaction and 172 indicated complete satisfaction. Another questionnaire was used in this study; the fatigue scale to measure fatigue in nurses who were employed. This questionnaire was designed by Rassouli et al. (28) in 2012, and consists of 20 statements with Likert scoring of 5 points (strongly agree, agree no opinion, disagree, and totally disagree). Each participant could receive from 0 to 100 points, 0 indicates the lowest fatigue and 100 indicates the highest fatigue. The content and formality of the material were evaluated by 19 specialist nurses, the internal consistency of the questionnaire was $\alpha=0.82$ and the correlation coefficient was two times the implementation of the test in the interval of 3 weeks, $r=0.88$. Its criterion validity was investigated by calculating the correlation coefficient of the scale with the "Visual Fatigue Scale" $(r=0.80)$. Structural validity through the exploratory factor analysis revealed the existence of 3 subscales of "cognitive-mental factor", "emotional-emotional factor," and "physical-behavioral factor". For assessing the validity of the questionnaire in the present study, 10 faculty members from the Faculty of Nursing and Midwifery of Shahid Beheshti University of Medical Sciences were asked to examine the content validity by reviewing the simplicity, clarity, and relevance. To check consistency reliability, the questionnaires were distributed among 20 nurses in two shifts. The test was taken twice with a twoweek interval. The correlation coefficient was $\mathrm{r}=0.70$ for the questionnaire of non-injectable medication errors, $\mathrm{r}=0.75$ for the questionnaire of injectable medication errors, $r=0.82$ for job satisfaction and for fatigue $r=0.88$. Cronbach's alpha coefficient was evaluated for measuring the internal consistency reliability of the questionnaires. The coefficient was calculated $\alpha=0.94$ for job satisfaction, $\alpha=0.92$ for fatigue, $\alpha=0.86$ for injectable medication error and $\alpha=0.87$ for non-injectable medication errors. Data was analyzed by SPSS version 17 . Independent-samples t-test was used to compare the mean of the two societies and the variance analysis was used to compare several societies. Also, the Pearson correlation coefficient for quantitative and qualitative variables was used. In order to do this study, a written letter was sent to the hospital authorities for permission to carry out the research. All the goals and stages of the research were announced to the participants. The participants were given the right to choose whether or not to participate in the research. All participants were notified of the restraint of all information.

\section{Results}

The findings showed that 170 nurses working in Shahid Sadoughi hospital in Yazd participated in this study whose demographic characteristics are illustrate in Table 1. According to results, most medication errors were injectable errors with the mean of 25.75 compared to non-injectable medication errors with the mean of 22.09. The largest number of errors $(32 \%)$ was 1-2 times, which mostly (52\%) occurred during night shifts. The mean of job satisfaction of nurses was $42.60 \%$ with a standard deviation of 17.44 . The findings showed that there was no significant relationship between job satisfaction and medication errors and with increased job satisfaction of nurses, the rate of errors was reduced, but this relationship was not statistically significant $(\mathrm{p}=0.214$ in non-injectable and $\mathrm{p}=0.057$ in injectable). The results also indicated that job satisfaction of women (43.94) was significantly greater than job satisfaction of men (37.07) $(\mathrm{p}=0.042)$. The average score of fatigue in nurses is $45.25 \%$ and standard deviation is 22.17. The findings showed that fatigue is not significantly associated with the type and amount of medication errors $(p=0.711$ in non- inject and $p=0.819$ in inject). Also, although fatigue and medical errors were more by men than women, the difference was not statistically significant $(p>0.05)$. The results show that fatigue in the contract of employment group is more than in other groups. Among the factors associated with non-injectable medication errors, only age was significantly related and by increasing age, the error was reduced $(p=0.037)$. Table 2 shows the correlation between non-injectable medication errors, intravenous and fatigue with demographic characteristics of the nurses participating in the study.

Table 1. Characteristics of the study participants

\begin{tabular}{|l|l|l|l|}
\hline Variable & $\mathrm{n}$ & $\%$ \\
\hline Gender & Male & 33 & 19 \\
\cline { 2 - 4 } & Female & 137 & 81 \\
\hline \multirow{3}{*}{ Marital Status } & Single & 58 & 34 \\
\cline { 2 - 4 } & Married & 110 & 65 \\
\cline { 2 - 4 } & Other & 60 & 35 \\
\hline \multirow{5}{*}{ Employment Status } & Contractual & 53 & 31 \\
\cline { 2 - 4 } & Official & 72 & 42 \\
\cline { 2 - 4 } & Agreement Project & 45 & 27 \\
\hline Total & & 170 & 100 \\
\hline
\end{tabular}


Table 2. Correlation between nurses' demographic characteristics with non-injectable medication errors, injectable medication errors, job satisfaction and fatigue

\begin{tabular}{|c|c|c|c|c|c|c|}
\hline Medication errors & Variables & Mean & SD & t-test & Pearson coefficient & $\mathrm{p}$-value \\
\hline \multirow[t]{8}{*}{ Non-injectable } & Female & 21.19 & 21.44 & \multirow[t]{2}{*}{-1.12} & & 0.264 \\
\hline & Male & 25.83 & 21.36 & & & \\
\hline & Married & 20.30 & 21.31 & \multirow[t]{2}{*}{-1.23} & & 0.219 \\
\hline & Single & 24.56 & 21.36 & & & \\
\hline & Job Satisfaction & 42.60 & 17.44 & & -0.096 & 0.214 \\
\hline & Fatigue & 45.25 & 22.17 & & -0.029 & 0.711 \\
\hline & Age & 33.62 & 6.55 & & -0.16 & 0.037 \\
\hline & Experience & 7.48 & 4.61 & & -0.136 & 0.078 \\
\hline \multirow[t]{8}{*}{ Injectable } & Female & 25.48 & 22.83 & \multirow[t]{2}{*}{-0.314} & & 0.194 \\
\hline & Male & 26.90 & 25.54 & & & \\
\hline & Married & 24.79 & 23.23 & \multirow[t]{2}{*}{-0.605} & & 0.546 \\
\hline & Single & 27.08 & 23.52 & & & \\
\hline & Job Satisfaction & 42.60 & 17.44 & & -0.146 & 0.057 \\
\hline & Fatigue & 45.25 & 22.17 & & 0.018 & 0.819 \\
\hline & Age & 33.62 & 6.55 & & -0.128 & 0.096 \\
\hline & Experience & 7.48 & 4.61 & & -0.095 & 0.217 \\
\hline
\end{tabular}

\section{Discussion}

This study aimed to investigate the correlation between job satisfaction and fatigue with medication errors in Shahid Sadooghi hospital in Yazd, Iran. Results showed that medication errors in Shahid Sadooghi hospital are fairly common. The result of this research is in agreement with the study by Taheri et al. (26). In their study on neonatal intensive care unit nurses, it showed that $37.8 \%$ of nurses had errors 1 to 2 times over 6 months (26). The results showed injectable errors greater than the mean non-injectable medication errors, which is aligned with Cheraghi et al. (10). They reported intravenous medication errors as $44.57 \%$ and oral medication errors as $19.14 \%$ (10). Also, in the race research, Mohamadnejad et al. (2009), on the four teaching hospitals in Tehran were students, most of intravenous medication errors reported (51.35\%) (29). However, Shams et al. (2012) showed that most medication errors related to oral medication errors $(57.7 \%)(30)$, so the results of this study are to the contrary. The difference between male and female nurses' job satisfaction was an additional finding of this study. Data analysis showed that the average of women's job satisfaction was higher than the average of men's job satisfaction. According to Joulaie et al. (2009), some factors such as negative attitude of society, nurses and other health care team members may result in reluctance to nursing, particularly among men. This influences their job satisfaction. It appears that multiple shifts and multiple simultaneous jobs are other factors of the reduction in men's job satisfaction compared to women's (7). However, Monjamed et al. (2004) found no statistically significant correlation between gender and job satisfaction (17), which is not in agreement with the results of this study. The findings showed that there was no significant relationship between job satisfaction and medication errors; however, with increased job satisfaction of nurses, the rate of errors was reduced, but this relationship was not statistically significant. In a similar study, Wilkins and Shields (2008) showed that 19\% of nurses believe that the main reasons of medication errors are working overtime, lack of human resource, lack of colleagues' support, and job insecurity (as indicators of job dissatisfaction). Medication errors reported by Wilkins and Shields were $18 \%$ in nurses with high job satisfaction and $28 \%$ in nurses with low job satisfaction but without statistically significant relationship between them (31). It can be said that despite the relationship between nurses' job satisfaction and the quality of care delivered to patients, sometimes increasing nurses' job satisfaction may lead to the reduction in the quality of care. Because, as job satisfaction increases, nurses prefer to take responsibility for planning patient care instead of taking care of patients, so patients' access to them becomes less (21). Chaudhury et al. showed in their study that inappropriate environmental factors cause the reduction in job satisfaction and consequently, the increase in medication errors (32). Also, in another study by Ghasemi et al. (2008), researchers found that workplace conditions were one of the factors affecting the incidence of medication errors (33), so the results of these studies are inconsistent. Findings of this study also showed an increased incidence of fatigue in medical errors of nurses, but the increase is not statistically significant. The study also showed that high workload, low numbers of staff, nurses, physical or mental fatigue, and prolonged working hours were the major cause for medical errors. In a similar study, Nikpeyma and Gholamnejad found that the major causes of medication errors were respectively, high labor costs $(70 \%)$, low staff (62\%), physical or mental fatigue (59\%) and high labor hours (58\%). So, fatigue is listed as the third reason for 
medication errors (34), which is in line with the results of this study. Holden et al. in a research on nurses at 6 units of a children's hospital, concluded that fatigue and a decrease in sufficient manpower caused job dissatisfaction among nurses. Finally, it was shown that there is a relationship between the workload of the nurses (both inside and outside the work environment) and medication errors (35). The study by Mitsis et al. on 176 nurses also showed increased workload, distraction, fatigue and burnout in nurses were the most prevalent causes of medication errors (36). Therefore, boredom and burnout are the main reasons cited for medical errors that are inconsistent with the results of this study. It seems that one of the reasons for the lack of agreement in the results of various studies, is the frequency of medication errors in the various nursing methods on the one hand, and the difference in the fatigue assessment tools for nurses on the other hand. Self report has been identified as a good way to determine medication errors in nurses (37). But regarding nurses in Iran, it seems, the actual number of errors in the report itself, are skeptical. Also, in this study, the instrument used to determine the levels of nurse fatigue, the chronic fatigue measurement tool, makes it plausible that chronic fatigue has a direct impact on the incidence of medication errors and their devastating effects, as an intermediary or mediator of variables, put on medication errors. In determining the amount of medication errors, findings showed that the average frequency of errors in this study was 1 to 2 times, and $28 \%$ believed that they had not made any errors during the last 6 months. These study findings are in agreement with Taheri's (2011) which indicated that, $37.8 \%$ of nurses have made 1 to 2 errors during the past 6 months on the neonatal intensive care unit (26). Hellstrom et al. (2012) showed that $50 \%$ of patients are at risk of medication errors, such that over $23 \%$ of patients, once, $11 \%$ of patients, twice, and $13 \%$ of patients, 3 to 9 times have experienced such errors (38). According to research, medication errors seem really prevalent. Findings suggest that the average of injectable medication errors $(26 \%)$ is more than the average of non-injectable medication errors $(23 \%)$, which is in agreement with the study of Cheraghi et al. (10). They reported intravenous medication errors at $57.44 \%$ and oral medication errors at $14 / 19 \%(10)$. In another research, Panjoveini (2006) stated that the highest rate of medication errors occurs in intramuscular injections (24.3\%), intravenous injections $(18.3 \%)$, and oral medications (15.2 percent) (39). Shams et al. (2012) believe that the most common medication errors are oral medication errors $(57.7 \%)$, because some medications are taken simultaneously (30), so the results are contrary to the ones of this study. The findings indicate a significant relationship between the age of nurses and medication errors, especially non-injectable medication errors, so that medication errors are more prevalent in younger and more novice nurses. As Heydari et al. discuss, nurses with lower work experience are slower than nurses with higher work experience. Multiple job responsibilities require time and speed that can lead to additional medication errors (40). This result is in agreement with the results of Shams et al. (2012). They showed that the prevalence of medication errors in ages 20 to 30 is higher (30). However, Keshk and Abdel-Moneem (2012), as well as Toruner and Uysal (2009) mentioned that there is no significant relationship between age and medication errors $(41,42)$, so the results are not consistent with this study. Since, in this study, data of medication errors were collected self-reportedly, the answers to the questionnaires might have been influenced by several factors such as fear of revealing personal data, punishment, change in colleagues' behaviour, overestimated self-assessment and downgrading. Although the researcher tried to ensure the nurses the confidentiality of data, many refused to mention the name of the wards such that the amount of medication errors could not be measured separately for each ward.

\section{Conclusions}

As it has been mentioned, results showed no significant relationship between job satisfaction and fatigue with medication error. Nurses' age was the most significant variable that associated with medication errors between demographic characteristics, but no significant relationship was found between others, such as gender, marital status, and type of employment and mean of medication errors. The results of this study can be used to improve the knowledge of nurses, carry out further research, clinical care and ultimately, help nursing managers to eliminate these factors. For future studies, it is suggested to review the barriers to reporting medication errors and provide solutions to reduce these barriers, to investigate the severity of medication errors and provide solutions to address them. Despite the fact that self-report is the best way of collecting data related to drug errors, it seems to be that the nurses did not mention all the cases in reporting their errors. Therefore, it seems necessary to design and measure psychometric instruments for measuring medication errors in nurses. A further research on medication errors and on nursing society may be a suitable route for future research in this subject.

\section{Acknowledgments:}

This study is a part of a master's thesis project of the Nursing and Midwifery Research Council in Shahid Beheshti University of Medical Sciences. Researchers thereby declare their gratitude and appreciation to all those who have assisted in this research. 


\section{Conflict of Interest:}

There is no conflict of interest to be declared.

\section{Authors' contributions:}

All authors contributed to this project and article equally. All authors read and approved the final manuscript.

\section{References:}

1) Valentin A, Capuzzo M, Guidet B, Moreno R, Metnitz B, Bauer P, et al. Errors in administration of parenteral drugs in intensive care units: multinational prospective study. BMJ. 2009; 338: b814. doi: 10.1136/bmj.b814. PMID: 19282436, PMCID: PMC2659290.

2) Hashemi F, Nasrabadi AN, Asghari F. Factors associated with reporting nursing errors in Iran: a qualitative study. BMC Nurs. 2012; 11: 20. doi: 10.1186/1472-6955-11-20. PMID: 23078517, PMCID: PMC3534596.

3) Ghaleb MA, Barber N, Dean Franklin B, Wong ICK. What constitutes a prescribing error in pediatrics? Qual Saf Health Care. 2005; 14: 352-7. doi: 10.1136/qshc.2005.013797. PMID: 16195569, PMCID: PMC1744084.

4) Bakken S, Cook SS, Curtis L, Desjardins K, Hyun S, Jenkins M, et al. Promoting patient safety through informatics-based nursing education. Promoting patient safety through informatics-based nursing education. Int J Med Inform. 2004; 73: 581-9. doi: 10.1016/j.ijmedinf.2004.04.008. PMID: 15246038.

5) Sheldon T. Dutch study shows that $40 \%$ of adverse incidents in hospital are avoidable. BMJ. 2007; 334(7600): 925. doi: 10.1136/bmj.39199.498137.DB. PMID: 17478822, PMCID: PMC1865418.

6) Reddy LKV, Modi AG, Chaudhary B, Modi V, Patel M. Medication errors - a case study. Journal of the Academy of Hospital Administration. 2009; 21: 28-34.

7) Joolaee S, Hajibabaee F, Peyrovi H, Haghani H. The occurrence and reporting of Medication errors and its relation to nurses working in hospitals in Iran University of medical sciences. Journal of Medical Ethics and History of Medicine. 2009; 3(1): 65-76.

8) hajibabaiee $\mathrm{F}$, jolaee $\mathrm{S}$, payravi $\mathrm{H}$, hagani $\mathrm{H}$. The relationship of medication errors among nurses with some organizational and demographic characteristics. IJNR. 2011; 6(20): 83-92.

9) Covell CL, Ritchie JA. Nurses' responses to medication errors suggestions for the development of organizational strategies to improve reporting. J Nurs Care Qual. 2009; 24(4): 187-297. doi: 10.1097/NCQ.0b013e3181a4d506. PMID: 19755878.

10) Cheraghi MA, Nikbakht Nasrabadi A, Mohamadnejad A, Salari A, Ehsani Kouhi Kheyli SR. Medication errors among nurses in intensive care units (ICU). J Mazandaran Univ Med Sci. 2011; 21(1): 115-9.

11) Mayo AM, Duncan D. Nurse perceptions of medication errors: what we need to know for patient safety. J Nurs Care Qual. 2004; 19(3): 209-17. doi: 10.1097/00001786-200407000-00007. PMID: 15326990.

12) Baghcheghi N, Kouhestani HR. Nursing students' errors in the preparation and administration of intravenous drugs. Strides in development of medical education. 2008; 5(1): 43-9.

13) Simonsen BO, Johansson I, Daehlin GK, Osvik ML, Farup PG. Medication knowledge, certainty, and risk of errors in health care: a cross-sectional study. BMC Health Services Research. 2011; 11(175): 1-9. doi: 10.1186/1472-6963-11-175.

14) Murrells T, Robinson S, Griffiths P. Job satisfaction trends during nurses' early career. BMC Nurs. 2008; 7(7): 1-13. doi: 10.1186/1472-6955-7-7. PMID: 18534023, PMCID: PMC2435528.

15) Darvish H. Study of effective factors of employee's job satisfaction (research-analytical approach). Organizational culture management. 2007; 16(5): 117-40.

16) Mirzabeigi Gh, Salemi S, Sanjari M, Shirazi F, Heidari Sh, Maleki S. Job Satisfaction among Iranian Nurses. Journal of Faculty of Nursing and Midwifery, Tehran University of Medical Sciences. 2009; 15(1): 49-59.

17) Monjamed Z, Ghorbani T, Mostofian F, Oveissipour R, Mahmoudi M. A nationwide study of level of job satisfaction of nursing personnel in Iran. Hayat. 2004; 10(23): 39-48.

18) Iliopoulos E, Priporas CV. The effect of internal marketing on job satisfaction in health services: A pilot study in public hospitals in Northern Greece. BMC Health Serv Res. 2011; 11(261): 1-8. doi: 10.1186/1472-6963-11-261. PMID: 21981753 PMCID: PMC3200181.

19) Al-hussami M. A study of nurses' job satisfaction: the relationship to organizational commitment, perceived organizational support, transactional leadership, transformational leadership, and level of education. European Journal of Scientific Research. 2008; 22(2): 286-95.

20) Bagheri Sh, Kousha A, Janati A. Factors Affecting Nurses' Job Satisfaction: a Systematic Survey. Hakim. 2012; 15(2): 130-9. 
21) Peltier J, Dahl A. The relationship between employee satisfaction and hospital patient experiences. NORTHWESTERN UNIVERSITY. April 2009. Available from: http://www.infonow.com/typo3conf/ext/p2wlib/pi1/press2web/html/userimg/FORUM/Hospital\%20Study\%20 -

Relationship\%20Btwn\%20Emp.\%20Satisfaction\%20and\%20Pt.\%20Experiences.pdf

22) Franklin N. Identifying medication documentation errors using handwritten versus pre-printed ICU flowcharts. Australian journal of advanced nursing. 2010; 29(3): 30- 9.

23) Sanjari M, Shirazi F, Heydari Sh, Maleki s, Saalemi S. Association of sleep status with occupational injuries among nurses. Journal of Nursing and Midwifery, Iran University of Medical Sciences. 2009; 22(61): 32-41.

24) Rogers AE, Hwang WT, Scott LD, Aiken LH, Dinges DF. The working hours of hospital staff nurses and patient safety. Health Affairs. 2004; 23(4): 202-12. doi: 10.1377/hlthaff.23.4.202. PMID: 15318582.

25) Wakefield BJ, Uden-Holman T, Wakefield DS. Development and validation of medication administration error reporting survey. Advance in Patient Survey. 2005; 4(12): 475-89.

26) Taheri E, Norian M, Rassouli M, Kavoosi A. The study of type and amount of medication errors in neonatal intensive care units and neonatal units. Iranian Journal of Critical Care Nursing. 2011: 100.

27) Sommerfeldt V. An identification of factors influencing police workplace motivation. 2010. Available from: http://www.eprints.qut.edu.au/34460/.

28) Rassouli M, Zandieh S, Noorian M, Zayeri F. Oncology nurses fatigue scale design and psychometric. Uremia journal of nursing and midwifery. 2012; 10(5): 640-7.

29) Mohamadnejad E. Hojati H, Sharifnia H, Ehsnai R. Survey of frequency and type of medication error among nursing students in four teaching hospitals in Tehran. Journal of Medical Ethics and History of Medicine. 2009: 60-9.

30) Shams Sh, Bagheria F, Faizi A, Baghaei R, Hashemlo L. Frequency of medication errors and its reporting to self-report by nurses in hospitals of Khoy city. Journal of Nursing and Midwifery of Uremia. 2012: 10(4): 721-6.

31) Wilkins K, Shields M. Correlates of medication error in hospitals. Component of Statistics Canada. 2008; 19(2): 1-13.

32) Chaudhury H, Mahmood A, Valente M. The effect of environmental design settings. Environment and Behavior. 2009; 41(6): 754-78.

33) Ghasemi F, Valizadeh F, Momen Nasab M. Review comments and knowledge of nurses regarding medication errors and ways to prevent the teaching hospitals in Khorram Abad. Lorestan University of Medical Sciences, School of Nursing. 2008; 10(2): 55-63.

34) Nikpeyma N, Gholamnejad H. Reasons for medication errors in nurses' views. Journal of Nurse Midwifery. 2009; 64(19): 18-24.

35) Holden RJ, Scanlon MC, Patel NR, Kaushal R, Escoto KH, Brown RL, et al. A human factors framework and study of the effect of nursing workload on patient safety and employee quality of working life. BMJ Quality Safety. 2011; 20: 15-24. doi: 10.1136/bmjqs.2008.028381.

36) Mitsis D, Kelesi M, Kapadohos T. Factors which affect the occurrence of nursing errors in medication administration and the errors' management, Rostrum of Asclepius. 2012; 11(2): 293-312.

37) Murff HJ, Patel VL, Hripcsak G, Bates DW. Detecting adverse events for patient safety research: a review of current methodologies. J Biomed Inform. 2003; 36: 131-43. doi: 10.1016/j.jbi.2003.08.003. PMID: 14552854.

38) Hellstrom LM, Bondesson A, Hoglund P, Eriksson T. Errors in medication history at hospital admission: prevalence and predicting factors. BMC Clin Pharmacol. 2012; 12(9): 1-9. doi: 10.1186/1472-6904-12-9. PMID: 22471836, PMCID: PMC3353244.

39) Panjevini S. Investigation of the rate and type of medication errors of nurses in Sanandaj hospitals. Iran Journal Nurses Research. 2006; 1(1): 59-64.

40) Heydari H, Kamran A, Pirzadeh A. Assessment of nurses' perceived barriers and behaviors to reporting medication errors in hospitals of Lorestan university of medical sciences, Iran. Health Systems Research. 2012; 8(5): 1-8.

41) Keshk LI, Abdel-Moneem DS. Effect of nurses' work hours and fatigue on occurrence of medication errors in ICU and medical oncology Unit -Cairo University. Life Science Journal. 2012; 9(3): 347-55.

42) Toruner EK, Uysal G. Causes, reporting, and prevention of medication errors from a pediatric nurse perspective. Australian Journal of Advanced Nursing. 2009; 29(4): 28-35. 